\title{
Liver Injury Resolution after Ruxolitinib Treatment in Newly Diagnosed Primary Myelofibrosis
}

Pinto GA ${ }^{1}$, García-Gutiérrez V ${ }^{\mathfrak{} *}$, Tellez $L^{2}$, PirisVillaespesa $M^{1}$, Herrera Puente $P^{1}$, Page $I^{1}$, Ruiz FA $^{1}$, Velázquez $\mathrm{K}^{1}$, Romio $\mathrm{E}^{3}$ and López $\mathrm{J}^{1}$

${ }^{1}$ Department of Hematology, Hospital Universitario Ramón Y Cajal, University of Alcalá de Henares, Spain ${ }^{2}$ Department of Gastroenterology and Hepatology, Hospital Universitario Ramón Y Cajal, University of Alcalá, Spain

${ }^{3}$ Department of Pathology, Hospital Universitario Ramón Y Cajal, University of Alcalá de Henares, Spain

*Correspondling author: García-Gutiérrez V, Department of Hematology, Hospital Ramón Y Cajal, Carretera Colmenar Viejo km 9.100, Madrid, Spain

Received: J une 09, 2017; Accepted: July 03, 2017;

Published: July 24, 2017

\section{Abstract}

Ruxolinitib is an approved treatment agent for patients with mylofibrosis that has proven efficacy in reducing splenomegaly, constitutional symptoms, weight loss, levels of cytokines driving systemic inflammation, and anti-pruritus effect. Recent evident suggest that the benefits of ruxolitinib go beyond these previously reported. We describe a case that shows a complete resolution of severe liver impairment secondary to myelofibrosis damage.

Keywords: Ruxolitinib; Myelofibrosis; Extramedullary hematopoiesis; Portal hypertension

\section{Abbreviations}

MF: Myelofibrosis; EMH: Extramedullary Hematopoiesis; PB: Peripheral Blood; MPN: Myeloproliferative Neoplasms

\section{Introduction}

Myelofibrosis (MF) is a BCR-ABL1-negative chronic myeloproliferative disorder characterized by variable degrees of bone marrow fibrosis, ineffective hematopoiesis, and extramedullary hematopoiesis (EMH). EHM in the gastrointestinal tract may result in hepatomegaly, abnormal liver function tests, coagulopathy, intrahepatic venous obstruction, abdominal pain, massive splenomegaly and portal hypertension. The latter may manifest with ascites (also as a consequence of EMH), esophageal or gastric varices, and splanchnic vein thrombosis. Ruxolinitib is an approved treatment agent for patients with MF that has proven efficacy in reducing splenomegaly, constitutional symptoms and weight loss, levels of cytokines driving systemic inflammation, and also has an anti-pruritus effect $[1,2]$. The management of other disease-related complications, such as portal hypertension, bleeding or thrombosis, are specific for each condition. Nevertheless, there is recent evidence that other complications of MF could respond to JAK-inhibitor therapy.

\section{Case Presentation}

A 49-year-old female patient, with an unremarkable past medical history, was admitted on suspicion of severe liver failure. She complained of a 2 months history of diffuse abdominal pain associated with distention, weight loss and progressive jaundice. Clinical examination revealed severe splenomegaly, ascites and cachexia. Additional investigations showed hemoglobin: $8.3 \mathrm{gr} / \mathrm{dL}$ (transfusion dependency), total leucocyte count: $7560 / \mathrm{mm}^{3}$, platelet count: 291.000/ $\mathrm{mm}^{3}$, total bilirrubin: $35 \mathrm{mg} / \mathrm{dL}$, alkaline phosphatase: 449 $\mathrm{U} / \mathrm{L}$, aspartate aminotransferase: $70 \mathrm{U} / \mathrm{L}$, alanine aminotransferase: $66 \mathrm{U} / \mathrm{L}$, creatinine: $1.2 \mathrm{mg} / \mathrm{dL}$. Abdominal ultrasound examination demonstrated portal hypertension, chronic portal vein thrombosis, and confirmed splenomegaly and ascites. Ascitic fluid examination showed a serum ascites albumin gradient $>1.1$, and was negative for malignant cells and microbiological cultures. With suspicion of gastrointestinal bleeding, an upper endoscopy was performed, and large varices were detected and treated with non selective beta-blockers and band ligation. Viral, autoimmune, metabolic and alcoholic liver diseases were ruled out. Histopathological examination of a liver biopsy showed venous congestion and nonspecific post-central obstruction, without evidence of cirrhosis. Computed tomography confirmed portal cavernomatosis and Magnetic resonance showed signs of portal colangiopathy. A subsequent endoscopy retrograde colangiopancreatography confirmed proximal biliary obstruction requiring sphincterotomy and biliary stenting, but only with mild improvement of bilirrubin levels.

Given the absence of a definite diagnosis, a second liver biopsy was performed, which bore a preserved architecture with evidence of cholestasis and inflammation and absence of hepatic ductopenia or fibrosis. Areas of EMH were found within the hepatic parenchyma (Figure 1A and 1B). The patient was referred to the Hematology department for assessment. A peripheral blood (PB) film examination revealed a leukoerythroblastic reaction, and $\mathrm{PB}$ immunophenotyping was unremarkable. Bone marrow aspiration was dry and the trephine biopsy bore idiopathic MF with 3\% of blasts, dysplastic megakaryocytes and mild reticulin fibrosis. Cytogenetic study showed a normal caryotype. Molecular testing resulted positive for JAK2 V617F mutation (34\%). The patient was classified as intermediate-2 according to the DIPSS-plus score due presence of constitutional symptoms related to myelofibrosis, anemia with transfusion dependency, absent of blasts in peripheral blood, normal caryotype and normal platelets count. The patient started ruxolitinb $15 \mathrm{mg}$ BID and suffered numerous complications during therapy, including: refractory ascites requiring repeated paracentesis, spontaneous bacterial peritonitis caused by methicillin-resistant Staphylococcus 


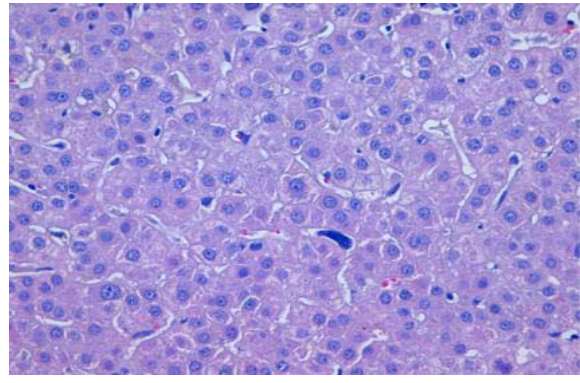

Figure 1A: Hematoxylin \& eosin stain with liver tissue preserved with signs of cholestasis, cytolytic focal activity and presence of intrasinusoidal megakaryocytes suggestive of extramedullar hematopoiesis.

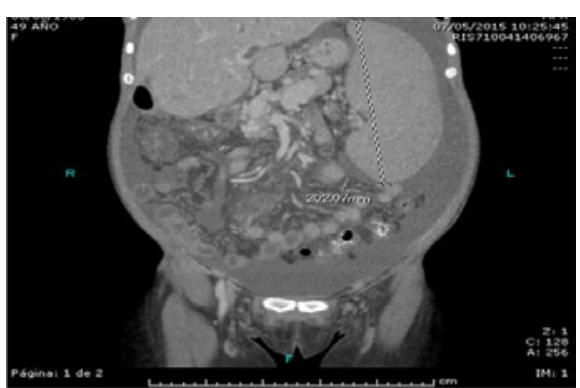

Figure 1B: Magnetic resonance imaging shows splenic enlargement, ascites, and chronic portal vein thrombosis.

aureus, Klebsiella pneumoniae, Pseudomona aeruginosa and Candida albicans, and bacteremia caused by Enterococcus faecium, and acute renal failure. The patient received multiple lines of antibiotic therapy and therapeutic paracentesis until complete resolution of the bacterial complications was achieved.

Promptly, after commencing ruxolitinib treatment, a progressive improvement in total bilirrubin levels was observed: ranging from an initial peak of $14 \mathrm{mg} / \mathrm{dL}$ to complete normalization after five months. Additionally, a complete recovery of the liver profile was also noted, as well as a moderate reduction in the creatinine level within a few days (Figure 2A). During the course of treatment, dose adjustment of ruxolitinib to $10 \mathrm{mg}$ BID was required due to liver impairment. Transfusional requirements were also reduced after starting ruxolitinib, maintaining acceptable hemoglobin and platelets counts (Figure 2B). With regards to constitutional symptoms such as malaise, weight loss or fatigue, the patient referred significant amelioration in the subsequent months, however the splenomegaly and ascites did not show any significant improvement even after 1 year of treatment, possibly due to the chronic portal thrombosis.

At the moment this manuscript was written the patient continues taking ruxolitinib $10 \mathrm{mg}$ BID with good performance status (ECOG 1), transfusion independent with no blasts increase in peripheral blood. The spleen size remained at last follow up without changes $(20 \mathrm{~cm})$. Bone marrow biopsy was performed 6 months after starting ruxolitinib, and it revealed an increase of erythroid cell hyperplasia, reduction of bone morrow fibrosis, without an advance in blasts percentage or additional changes in caryotype. DIPPS plus score was recalculated, although the patient is not transfusion depended any more, the patient continues being classified as intermediate-II, and

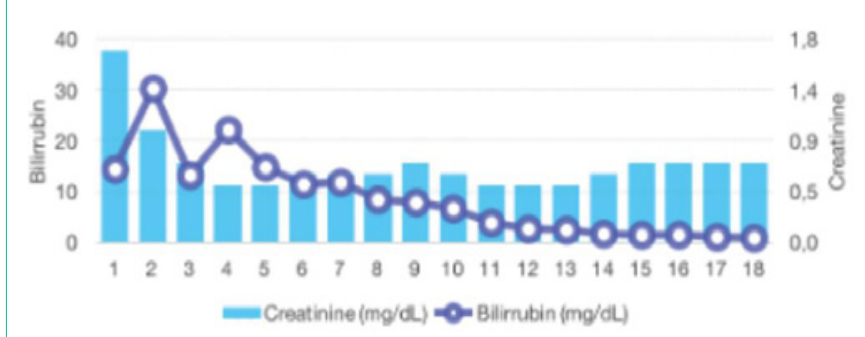

Figure 2A: Changes in total bilirubin and creatinine. Notice the initial elevation of bilirubin after starting ruxolitinib, and then, stabilization and declined due to dose adjustment.

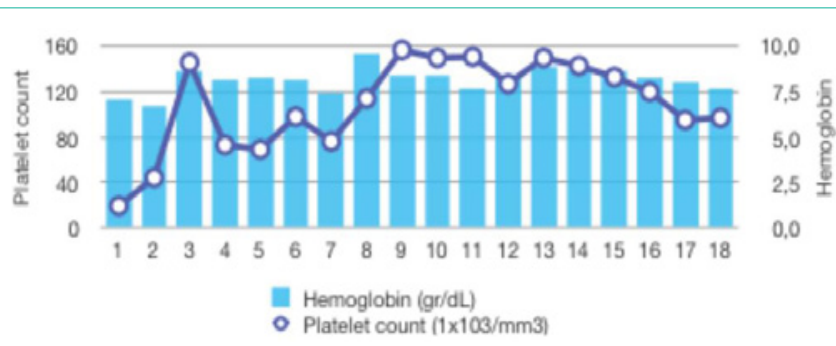

Figure 2B: Stabilization of hemoglobin and platelets counts after starting ruxolitinib.

therefore has been considered for bone marrow transplantation.

\section{Discussion}

We herein present a case of MF, with a difficult diagnostic process, reached by detection of EMH in the liver, with a significant clinical response to JAK1/2 inhibition by ruxolitinib. The clinical benefit attributed to ruxolitinib has been studied in some randomized controlled clinical trials, in which it was associated with a marked and durable reduction in splenomegaly and disease-related symptoms. It was also found to improve patient's functional capacity and overall quality of life [3]. However, other studies have suggested that the benefits of ruxolitinib go beyond these previously reported.

Recent evidence suggests that ruxolitinib may enhance normal hematopoiesis in other myeloproliferative neoplasms (MPN) by suppressing the malignant clone [4,5]. In fact, this effect is evidenced by the reduction of EMH in different organs secondary to MF caused by JAK-1/2 inhibitor. Maccaferri, et al. described a patient with pulmonary EMH who presented a decrease in dyspnea, cough, and oxygen requirements as well as improvement in pulmonary function tests and ground glass opacities during ruxolitinib therapy [6]. Koschmieder and colleagues reported a patient with reduction and stabilization of esophageal varices, attributed to a decrease in portal-vein hypertension [7]. Rajasekarn, et al, describe a case of MFN-related glomerulopathy in a patient recently diagnosed with primary MF with a significant improvement in kidney function upon successful therapy of MF with ruxolitinib [8].

In this case, we confirm a complete resolution of severe liver impairment with no effect on portal thrombosis, attributed to improvement of liver EMH by ruxolitinib. However, complete disappearance of EMH cannot be confirmed because no further liver biopsies were performed after ruxolitinib was started, and therefore, complete resolution of liver impairment was probably influenced 
by other factors as sepsis resolution or the diminish of portal hypertension as previously described [7]. The spleen size and ascites remained unchanged possibly due to the coexistence of chronic portal thrombosis, but it is feasible that ruxolitinib could also improve these associated complications in other clinical circumstances. We have also have shown how ruxotinib treatment is feasible by adjusting dose in patients with bad performance status (severe liver and kidney failure), severe haematological toxicities and concomitants infections. This is the first case reported in the literature of correction of liver impairment attributed to ruxolitinib in MF patients.

In summary, this case highlights the potential clinical benefits of ruxolitinib in others organs, such as the liver by decreasing EMH. Taking into account other recently published clinical reports, it seems reasonable to consider the addition of others indications for ruxolitinib, although more evidence is required in order to support this affirmation.

\section{References}

1. Verstovsek S, Mesa RA, Gotlib J, Levy RS, Gupta V, DiPersio JF, et al. A double-blind, placebo-controlled trial of ruxolitinib for myelofibrosis. $\mathrm{N}$ Engl J Med. 2012; 366: 799-807.
2. Harrison C, Kiladjian JJ, Al-Ali HK, Gisslinger H, Waltzman R, Stalbovskaya $V$, et al. JAK inhibition with ruxolitinib versus best available therapy for myelofibrosis. N Engl J Med. 2012; 366: 787-798.

3. Harrison CN, Vannucchi AM, Kiladjian JJ, Al-Ali HK, Gisslinger H, Knoops $\mathrm{L}$, et al. Long-term findings from COMFORT-II, a phase 3 study of ruxolitinib vs best available therapy for myelofibrosis. Leukemia. 2016; 30: 1701-1707.

4. Vannucchi AM, Kiladjian JJ, Griesshammer M, Masszi T, Durrant S Passamonti $F$, et al. Ruxolitinib versus standard therapy for the treatment of polycythemia vera. N Engl J Med. 2015; 372: 426-435.

5. Bryan J, Verstovsek S. Overcoming treatment challenges in myelofibrosis and polycythemia vera: the role of Ruxolitinib. Cancer Chemother Pharmacol. 2016; 77: 1125-1142.

6. Maccaferri M, Leonardi G, Marasca R, Colaci E, Paolini A, Soci F, et al. Ruxolitinib for pulmonary extramedullary hematopoiesis in myelofibrosis. Leuk Lymphoma. 2014; 55: 2207-2208.

7. Koschmieder S, Koppelle A, Seifert H. Ruxolitinib for myelofibrosis. N Engl J Med. 2012; 366: 2031-2032.

8. Rajasekaran A, Ngo TT, Abdelrahim M, Glass W, Podoll A, Verstovsek S, et al. Primary myelofibrosis associated glomerulopathy: significant improvement after therapy with ruxolitinib. BMC Nephrol. 2015; 16: 121.
Ann Hematol Oncol - Volume 4 Issue 8 - 2017

ISSN : 2375-7965 | www.austinpublishing group.com

García-Gutiérrez et al. (C) All rights are reserved
Citation: Pinto GA, García-Gutiérrez V, Tellez L, Piris-Villaespesa M, Herrera Puente P, Page I, et al. Liver Injury Resolution after Ruxolitinib Treatment in Newly Diagnosed Primary Myelofibrosis. Ann Hematol Oncol. 2017; 4(8): 1163. 